\title{
Treatment of Thoracolumbar Spinal Fracture Accompanied by Diffuse Idiopathic Skeletal Hyperostosis Using Transdiscal Screws for Diffuse Idiopathic Skeletal Hyperostosis: Preliminary Results
}

\author{
Hisanori Ikuma ${ }^{1}$, Shinichiro Takao ${ }^{2}$, Yoichi Inoue ${ }^{1}$, \\ Tomohiko Hirose ${ }^{1}$, Keitaro Matsukawa ${ }^{3}$, Keisuke Kawasaki ${ }^{1}$ \\ ${ }^{1}$ Department of Orthopaedic Surgery, Kagawa Prefectural Central Hospital, Takamatsu, Japan \\ ${ }^{2}$ Department of Orthopaedic Surgery, Graduate School of Medicine, Dentistry, and Pharmaceutical Science, Okayama University, Okayama, Japan \\ ${ }^{3}$ Department of Orthopaedic Surgery, National Hospital Organization, Murayama Medical Center, Musashimurayama, Japan
}

Study Design: This retrospective case series enrolled 13 patients who underwent posterior fixation with both transdiscal screws for diffuse idiopathic skeletal hyperostosis (TSDs) and pedicle screws (PSs) to treat spinal injury accompanied by diffuse idiopathic skeletal hyperostosis (DISH).

Purpose: To describe the usefulness, feasibility, and biomechanics of TSD.

Overview of Literature: Vertebral bodies accompanied by DISH generally have lower bone mineral density than normal vertebral bodies because of the stress shielding effect. This phenomenon tends to makes screw fixation challenging. To our knowledge, solutions for this issue have not previously been reported.

Methods: Patients were assessed using the data on surgical time, estimated intraoperative blood loss, mean number of stabilized intervertebral segments, number of screws used, perioperative complications, union rate, and the three-level Euro0ol five-dimensional questionnaire (E05D-3L) score at the final follow-up. The Hounsfield unit (HU) values of the screw trajectory area, and the actual intraoperative screw insertion torque of TSDs and PSs were also analyzed and compared.

Results: The surgical time and estimated intraoperative blood loss were $165.9 \pm 45.5$ minutes and $71.0 \pm 53.4 \mathrm{~mL}$, respectively. The mean number of stabilized intervertebral segments was 4.6 \pm 1.0 . The number of screws used was $4.9 \pm 1.3$ for TSDs and $3.0 \pm 1.4$ for PSs. One death occurred after surgery. The union rate and E05D-3L scores were $100 \%$ and $0.608 \pm 0.128$, respectively. The HU value and actual intraoperative screw insertion torque of TSDs were significantly better than those of PSs $(p<0.001, p=0.033)$.

Conclusions: We were able to achieve stable surgical outcomes using the combination of TSDs and PSs. The HU value and actual intraoperative screw insertion torque were significantly higher for TSDs than for PSs. Based on these results, when treating thoracolumbar spinal fractures accompanied by DISH in elderly populations, the TSD could be a stronger anchor than the PS.

Keywords: Diffuse idiopathic skeletal hyperostosis; Endplate; Pedicle screw; Computed tomography imaging

Received Feb 29, 2020; Revised Mar 22, 2020; Accepted Apr 20, 2020

Corresponding author: Hisanori Ikuma

Department of Orthopaedic Surgery, Kagawa Prefectural Central Hospital, 2-1, Asahi-machi 1-chome, Takamatsu City, Kagawa 7600065, Japan

Tel: +81-87-811-3333, Fax: +81-87-802-1188, E-mail: i9ma9n@yahoo.co.jp 


\section{Introduction}

Diffuse idiopathic skeletal hyperostosis (DISH) is characterized by spinal fusion between the vertebral bodies, including the anterior and posterior elements, resulting from bone hyperplasia and involving the osteophytes and bone bridges that arise from the anterior and posterior elements of the vertebral bodies. Morphologically, this results in the spine appearing as a single pillar. Accordingly, spinal fracture in the ankylosing spine, such as DISH, results in a three-column injury that is likely to cause greater instability of the fracture site and is more likely to lead to spinal cord injury than spinal fracture in the normal spine. Therefore, early diagnosis of injury and rigid spinal fixation are recommended [1,2]. Although it is commonly thought that the bone mineral density of vertebral bodies in the spine with DISH is elevated because of osteophytes and bone bridges that develop outside of the vertebral bodies, stress shielding arising from the ankylosing spine can reduce the bone mineral density in the vertebral column [3]. Therefore, in order to solve this problem, we propose the new concept of a screw insertion technique for posterior spinal fixation. The goal of this technique was to secure rigid fixation of the screw by penetrating the two vertebral endplates of the vertebral body, even in the osteoporotic spine accompanied by DISH in elderly patients. We report the usefulness and preliminary clinical results of this screw insertion technique that is referred to as the insertion of a transdiscal screw for diffuse idiopathic skeletal hyperostosis (TSD) in this report.

\section{Materials and Methods}

\section{Patient population and clinical and biomechanical outcome analyses}

Total 212 patients who were admitted to Kagawa Prefectural Central Hospital for the treatment of thoracolumbar spine fracture from April 2016 through March 2018 were assessed retrospectively based on their medical records. Patients aged $\geq 65$ years with spinal fracture in the form of a three-column injury accompanied by DISH who underwent posterior fixation using the combination of a TSD and pedicle screw (PS) in the range of two vertebral bodies superior and inferior from the fracture level who could be followed up for at least 1 year postoperatively were enrolled. Thirteen patients were finally identified and investigated in this study. All surgeries in this series were performed by one senior spine surgeon. Bone grafting for the posterior or anterior portion and vertebroplasty were not performed in this series. We evaluated the patients' demographic data, including age, sex, injury level, preoperative Frankel grade, and mean follow-up period; surgery-related parameters of operative duration, estimated intraoperative blood loss, number of spinal segments stabilized, number of screws used, and perioperative complications were also recorded. The postoperative follow-up was performed once every 3 months. At the final follow-up, we examined patient status by assessing the Frankel grade, bone union rate, and health-related quality of life (three-level EuroQol five-dimensional questionnaire [EQ5D-3L]) [4]. Bone union was determined using computed tomography (CT) images (sagittal plane, coronal plane, and transverse plane) that were obtained once every 6 months, and the presence of bone union was determined when trabecular bone continuity and sufficient bone bridge formation were observed at the fracture site. To evaluate the degree of screw fixation, we measured the Hounsfield unit (HU) value of the screw trajectory using the method that Matsukawa et al. [5] recently reported. They reported that the fixation stability can be predicted by calculating the $\mathrm{HU}$ of the planned screw trajectory using preoperatively obtained CT data. The maximum intraoperative screw insertion torque was also calculated using an exclusive measuring instrument.

\section{Transdiscal screw for diffuse idiopathic skeletal hy- perostosis}

A transdiscal screw (TS) that passes through the vertebral endplate was first reported in 2003 by Minamide et al. [6] in a biomechanical study for L5/S spondylolisthesis. The TS construct was 1.6-1.7 times stronger than the conventional PS construct, thereby demonstrating the usefulness of TSs [6]; unlike TSs, TSDs are used for the osteoporotic spine accompanied by DISH occurring from the thoracic to the lumbar spine. The aim of TSD insertion is to achieve better screw fixation by passing through two vertebral endplates, even in patients who have an osteoporotic spine. The vertebral endplate can offer improved screw fixation because of the subchondral bone around the vertebral endplate. 


\section{Transdiscal screw for diffuse idiopathic skeletal hy- perostosis insertion technique}

The entry point of the TSD is just below the pedicle (toward the 5 o'clock position for the right pedicle and toward the 7 oclock position for the left pedicle) and can be confirmed in the anteroposterior view using fluoroscopy. Especially for the lumbar spine, the entry point of the TSD is around the accessory process. Regarding the trajectory of the TSD, the screw passes through the pedicle in a cranial direction to penetrate two vertebral endplates, including the cranial vertebral endplate of the vertebrae that the screw entered through and the caudal endplate of the cranially adjacent vertebral body (Fig. 1). The surgeon always needs to be cautious to avoid penetrating the caudal side of the pedicle causing the radiculopathy when making the screw trajectory in the pedicle. The length of the TSD is determined with the aim of penetrating two vertebral endplates as much as possible without penetrating the anterior surface of the vertebral body. We utilized a percutaneous pedicle screw (PPS) for the spinal implant to minimize surgical invasiveness in all study subjects. If a guidewire is prepared for screw insertion in advance, it can enable the PPS to pass through two vertebral endplates. We commonly used intraoperative CT navigation for TSD insertion because most patients with the complication of DISH tend to have severe spinal kyphosis and ossification surrounding the vertebral body, making it difficult to determine the entry point and trajectory for TSD insertion using only fluoroscopy. In the case of small amount of spinal kyphosis and ossification surrounding the vertebral body, TSD insertion under only fluoroscopy
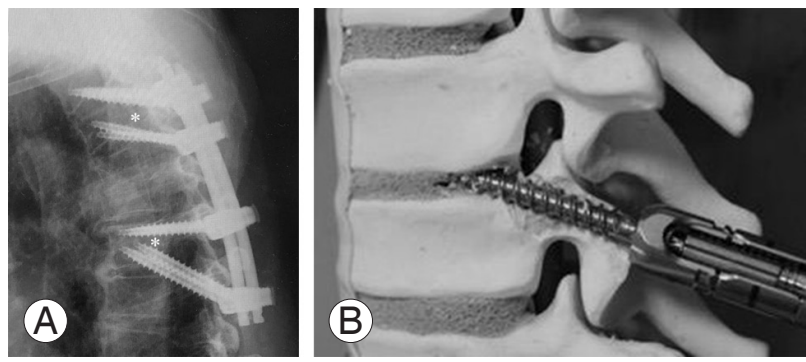

Fig. 1. (A) TSD: posterior fixation using the combination of TSDs and pedicle screws. Four screws are inserted into a single vertebral body (white asterisk). We think the stability of the local fixation can be increased by placing four of these screws. (B) A TSD inserted into a bone model. This photograph shows the trajectory of the TSD that penetrated two vertebral endplates after the screw passed through the pedicle and was eventually inserted into the vertebral body of the proximal adjacent vertebra. TSD, transdiscal screw for diffuse idiopathic skeletal hyperostosis. may be possible. The fluoroscopy is used for the lateral view for the verification of navigation error during the surgery in all study subjects.

\section{Hounsfield unit calculations}

Using CT image data, we calculated the $\mathrm{HU}$ value of the TSD and conventional PS trajectories. To perform image analysis on the preoperative CT scans, we used ZedView VEGA (LEXI Co. Ltd., Tokyo, Japan), wherein a virtual screw was placed according to the TSD and PS trajectories. The PS adopted a trajectory along the anatomical axis of the pedicle in order to reach the area just before the anterior wall of the vertebral body. The trajectory of the TSD passed through the pedicle to the posterior third of the cranial endplate of the vertebral body as well as the caudal endplate of the adjacent vertebral body to reach the area just before the anterior wall of the adjacent vertebral body. Thereafter, the screw trajectory was selectively extracted on the CT image, and the HU value of the screw trajectory was calculated as per to the sum of the mean HU values of each 1-mm segment along the screw axis of the

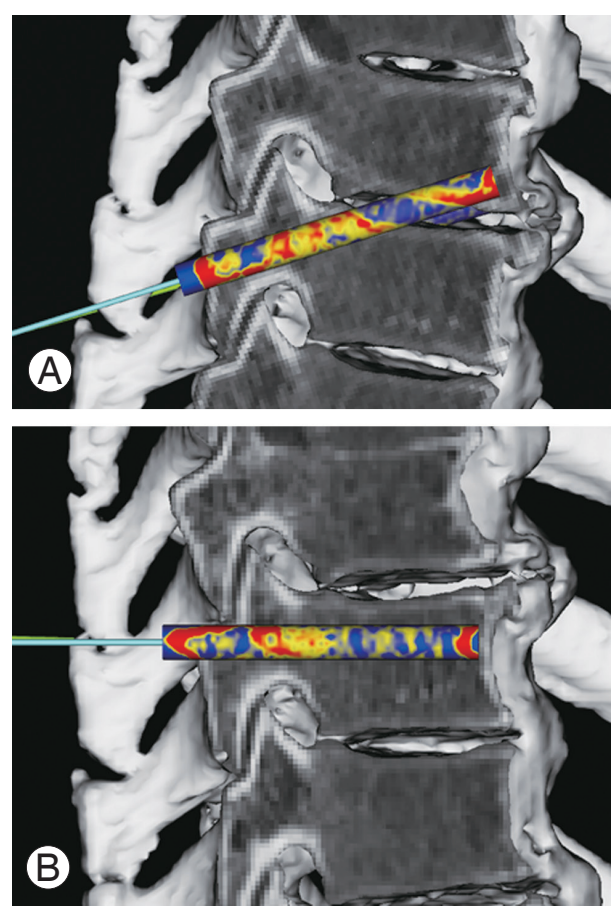

Fig. 2. A virtual screw trajectory was created on three-dimensional computed tomographic images; the mean $\mathrm{HU}$ values of each 1-mm segment from the insertion point to the tip of the screw were totaled, and the HU value of the screw trajectory was calculated. (A) Transdiscal screw for diffuse idiopathic skeletal hyperostosis trajectory, (B) Pedicle screws trajectory. HU, Hounsfield unit. 
largest circle that could be delineated within the pedicle (Fig. 2). Using this method, the HU measurements of the TSD and PS trajectories were calculated and compared, and the maximum screw diameter and maximum screw length capable of insertion were measured on CT images.

\section{Maximum intraoperative screw insertion torque measurements}

We measured and compared the maximum torque during TSD and PS insertion in the same patient in our case series. We used Precept (NuVasive Inc., San Diego, CA, USA) as the TSD that was inserted percutaneously in all patients. Torque measurements were performed using DTDK-CN500REV (Nakamura Mfg. Co. Ltd., Sagamihara, Japan). After a specialized screwdriver for measuring screw torque was attached to the screw, we measured the maximum torque using the continuous mode of the DTDK-CN500REV. We obtained measurements taken from the point of screw insertion until the screw head came into contact with the bone surface, and the screw was unable to rotate. The maximum value until this point was recorded as the maximum torque value. Furthermore, we calculated the mean screw diameter and the mean screw length of an actually inserted screw.

\section{Statistical analysis}

The screw torque test and $\mathrm{HU}$ were statistically examined using unpaired $t$-tests. A $p$-value $<0.05$ was considered statistically significant. In the present study, we used y-stat 2006 for Macintosh (Igakutosho shuppan Co. Ltd., Toda, Japan) to perform the statistical analyses.

We performed this investigation in accordance with our institutional guidelines that comply with international laws and policies (Institutional Review Board of Kagawa Prefectural Central Hospital, IRB approved no., 802). Informed consent was obtained from all individual participants included in this study.

\section{Results}

\section{Clinical outcomes}

The demographic data of the patients showed that their mean age was $81.2 \pm 7.5$ years; the study population included 11 men and two women. The mean follow-up duration was $17.08 \pm 4.4$ months, and the mean body mass index was $23.2 \pm 4.1 \mathrm{~kg} / \mathrm{m}^{2}$. The preoperative neurological status was classified as Frankel E for all the patients. The injury mechanism was most commonly minor trauma, such as a fall at same level, and there was a high incidence of injuries at the thoracolumbar junction (Table 1).

Table 1. Patient demographic data

\begin{tabular}{|c|c|c|c|c|c|c|}
\hline Patient no. & Age $(y r) / \operatorname{sex}$ & $\begin{array}{l}\text { Body mass index } \\
\left(\mathrm{kg} / \mathrm{m}^{2}\right)\end{array}$ & Mechanism of injury & Fractured level & Frankel grade & Follow-up (mo) \\
\hline 1 & $85 / F$ & 16.89 & Fall at same level & T11 (transvertebral) & $E$ & 23 \\
\hline 2 & $78 / \mathrm{M}$ & 25.76 & Traffic accident & T5/6 (transdiscal) & $E$ & 21 \\
\hline 3 & $65 / \mathrm{M}$ & 32.39 & Fall at same level & L4 (transvertebral) & $\mathrm{E}$ & 20 \\
\hline 4 & $87 / \mathrm{M}$ & 25.6 & Fall at same level & T9 (transvertebral) & $E$ & 19 \\
\hline 5 & $80 / \mathrm{M}$ & 20.31 & Fall from height & T5 (transvertebral) & $\mathrm{E}$ & 18 \\
\hline 6 & $86 / \mathrm{M}$ & 25.65 & Fall at same level & L2 (transvertebral) & $E$ & 18 \\
\hline 7 & $91 / F$ & 25.66 & Fall at same level & T10 (transvertebral) & $E$ & 17 \\
\hline 8 & $73 / \mathrm{M}$ & 22.94 & Fall from height & T12 (transvertebral) & $E$ & 15 \\
\hline 9 & $82 / \mathrm{M}$ & 19.71 & Fall at same level & T10 (transvertebral) & $E$ & 15 \\
\hline 10 & $86 / \mathrm{M}$ & 20.22 & Fall from height & T11/12 (transdisc) & $\mathrm{E}$ & 14 \\
\hline 11 & $91 / \mathrm{M}$ & 24.6 & Fall at same level & T11/12 (transdisc) & $E$ & 13 \\
\hline 12 & $75 / \mathrm{M}$ & 22.8 & Fall at same level & T7/8 (transdisc) & $E$ & 13 \\
\hline 13 & $77 / \mathrm{M}$ & 19.1 & Fall at same level & L1 (transvertebral) & $E$ & - \\
\hline
\end{tabular}

F, female; $\mathrm{M}$, male. 
Table 2. Surgical results in detail for all patients

\begin{tabular}{|c|c|c|c|c|c|c|c|c|c|c|}
\hline Patient no. & $\begin{array}{l}\text { Surgical time } \\
\text { (min) }\end{array}$ & $\begin{array}{l}\text { Blood loss } \\
\text { (mL) }\end{array}$ & $\begin{array}{l}\text { No. of segments } \\
\text { stabilized }\end{array}$ & $\begin{array}{l}\text { No. of screws } \\
\text { used }\end{array}$ & $\begin{array}{l}\text { No. of } \\
\text { TSDs }\end{array}$ & $\begin{array}{l}\text { No. of } \\
\text { PSs }\end{array}$ & Complications & $\begin{array}{c}\text { Union at } \\
\text { fracture site }\end{array}$ & $\begin{array}{l}\text { Frankel grade } \\
\text { (final FU) }\end{array}$ & $\begin{array}{l}\text { E05D-3L } \\
\text { score }\end{array}$ \\
\hline 1 & 254 & 120 & 4 & 8 & 4 & 4 & None & Yes & $E$ & 0.496 \\
\hline 2 & 126 & 17 & 3 & 8 & 4 & 4 & None & Yes & $E$ & 0.734 \\
\hline 3 & 257 & 200 & 4 & 8 & 4 & 4 & None & Yes & $E$ & 0.713 \\
\hline 4 & 180 & 110 & 5 & 8 & 6 & 2 & None & Yes & $E$ & 0.681 \\
\hline 5 & 116 & 30 & 5 & 8 & 6 & 2 & None & Yes & $\mathrm{E}$ & 0.474 \\
\hline 6 & 170 & 95 & 4 & 8 & 4 & 4 & None & Yes & $E$ & 0.496 \\
\hline 7 & 136 & 20 & 4 & 8 & 6 & 2 & None & Yes & $E$ & 0.386 \\
\hline 8 & 131 & 41 & 5 & 8 & 4 & 2 & None & Yes & $E$ & 0.729 \\
\hline 9 & 157 & 60 & 4 & 8 & 4 & 4 & None & Yes & $E$ & 0.598 \\
\hline 10 & 138 & 68 & 6 & 8 & 4 & 4 & None & Yes & $E$ & 0.521 \\
\hline 11 & 190 & 100 & 5 & 8 & 6 & 2 & None & Yes & $E$ & 0.729 \\
\hline 12 & 135 & 10 & 5 & 8 & 8 & 0 & None & Yes & $E$ & 0.734 \\
\hline 13 & 167 & 52 & 7 & 9 & 4 & 5 & Death & - & - & - \\
\hline
\end{tabular}

TSDs, PSs, pedicle screws; FU, follow-up; E05D-3L, three-level EuroQol five-dimensional questionnaire.

Table 3. Summary of the surgical results

\begin{tabular}{lc} 
Variable & Value \\
No. of TSDs & 13 \\
\hline Surgical time (min) & $165.9 \pm 45.5$ \\
\hline Intraoperative blood loss (mL) & $71.0 \pm 53.4$ \\
\hline No. of segments stabilized & $4.6 \pm 1.0$ \\
\hline No. of TSDs used & $4.9 \pm 1.3$ \\
\hline No. of PSs used & $3.0 \pm 1.4$ \\
\hline Perioperative complications & 1 (Postoperative death) \\
\hline Fusion rate (final follow-up, \%) & 100 \\
\hline EQ5D-3L (final follow-up) & $0.608 \pm 0.128$ \\
\hline
\end{tabular}

Values are presented as number or mean \pm standard deviation, unless otherwise stated

TSD, transdiscal screw for diffuse idiopathic skeletal hyperostosis; PSs, pedicle screws; E05D-3L, three-level Euro0ol five-dimensional questionnaire.

The mean surgical time and the mean estimated intraoperative blood loss were $165.9 \pm 45.5$ minutes and $71.0 \pm 53.4 \mathrm{~mL}$, respectively. The mean number of spinal segment levels stabilized was $4.6 \pm 1.0$, and the mean number of screws used was $8.0 \pm 0.2(4.9 \pm 1.3$ for the TSDs and $3.0 \pm 1.4$ for the PSs). The perioperative complications included one death caused by chronic heart failure 1 month after the surgery. However, no implant-related complications were observed during the follow-up. At the time of the final follow-up, the Frankel grade was E in all patients; all patients achieved bone union, and the mean EQ5D-
Table 4. HU values, screw diameters, and screw lengths of the TSD and PS trajectories on computed tomography images

\begin{tabular}{lccc} 
Variable & $\begin{array}{c}\text { TSD trajectory } \\
(\mathrm{n}=96)\end{array}$ & $\begin{array}{c}\text { PS trajectory } \\
(\mathrm{n}=96)\end{array}$ & $p$-value \\
\hline HU & $13,277 \pm 3,734$ & $9,114 \pm 3,331$ & $<0.001$ \\
\hline Screw diameter $(\mathrm{mm})$ & $5.7 \pm 0.9$ & $5.7 \pm 0.9$ & NS \\
\hline Screw length $(\mathrm{mm})$ & $50.6 \pm 4.0$ & $44.8 \pm 5.3$ & $<0.001$ \\
\hline
\end{tabular}

Values are presented as mean \pm standard deviation. Bold type shows statistical significance; $p$-value for the difference between the TSD trajectory and PS trajectory.

HU, Hounsfield unit; TSD, transdiscal screw for diffuse idiopathic skeletal hyperostosis; PSs, pedicle screws; NS, not significant.

$3 \mathrm{~L}$ score was $0.608 \pm 0.128$. Over the course of the followup, there was no clear correction loss, screw loosening, or screw pull-out (Tables 2, 3).

\section{Hounsfield unit values}

For the initial six patients (five men and one woman, with a mean age of $82.6 \pm 3.7$ years) in our series, we used preoperative CT to measure the HU values of the TSD trajectories (96 screws) and PS trajectories (96 screws) in 48 vertebral bodies (37 thoracic vertebrae and 11 lumbar vertebrae) with DISH factors. The mean $\mathrm{HU}$ value of the TSD trajectory was $13,277 \pm 3,734$, while the mean HU value of the PS trajectory was $9,114 \pm 3,331$, indicating a higher mean value for TSDs $(p<0.001)$. The maximum di- 
ameter for screw insertion was $5.7 \pm 0.9 \mathrm{~mm}$ for the TSDs and $5.7 \pm 0.9 \mathrm{~mm}$ for the PSs, indicating no significant difference. The maximum screw length was $50.6 \pm 4.0 \mathrm{~mm}$ for the TSDs and $44.8 \pm 5.3 \mathrm{~mm}$ for the PSs, indicating that a significantly longer TSDs could be inserted than the PSs $(p<0.001)$ (Table 4).

\section{Maximum intraoperative screw insertion torque}

We were able to measure the maximum torque at the time of screw insertion during surgery for 33 TSDs and 15 PSs. The mean maximum screw insertion torque was 175.3 $\pm 57.5 \mathrm{Nm}$ for the TSDs and $141.8 \pm 42.6 \mathrm{Nm}$ for

Table 5. The actual intraoperative screw insertion torque, and the diameter and length of the screws

\begin{tabular}{lccc} 
Variable & TSD $(\mathrm{n}=33)$ & PS $(\mathrm{n}=15)$ & $p$-value \\
Maximum screw insertion torque $(\mathrm{Nm})$ & $175.3 \pm 57.5$ & $141.8 \pm 42.6$ & 0.033 \\
Screw diameter $(\mathrm{mm})$ & $7.2 \pm 0.4$ & $7.3 \pm 0.4$ & $\mathrm{NS}$ \\
Screw length $(\mathrm{mm})$ & $54.7 \pm 2.5$ & $49.6 \pm 4.4$ & $<0.001$ \\
\hline
\end{tabular}

Values are presented as mean \pm standard deviation. Bold type shows statistical significance. p-value for the difference between the TSD trajectory and PS trajectory.

TSD, transdiscal screw for diffuse idiopathic skeletal hyperostosis; PSs, pedicle screws; NS, not significant. the PSs, indicating significantly higher values for TSDs $(p=0.033)$. The screw diameter was $7.2 \pm 0.4 \mathrm{~mm}$ for TSDs and $7.3 \pm 0.4 \mathrm{~mm}$ for PSs, indicating no significant difference. However, the screw length was $54.7 \pm 2.5 \mathrm{~mm}$ for TSDs and $49.6 \pm 4.4 \mathrm{~mm}$ for PSs, indicating the insertion of significantly longer TSDs $(p<0.0001)$ (Table 5).

\section{Case presentation}

The subject was a 78-year-old man (patient number 2) who was injured after being hit by a compact car while walking. He had severe back pain at the first visit. Disc space opening was found at T5/6 on CT scan. The thoracic spine, including the injury level, had extensive DISH cranially and caudally, and the amount of trabecular bone in the anterior to middle column around the fracture site was significantly reduced. Additionally, magnetic resonance imaging (MRI) revealed intensity changes in the disk space at T5/6 and around the posterior elements at the same level. It was assumed that a three-column injury was present in the T5/6 area. Furthermore, at the injury level, spinal canal stenosis was found on MRI; however, no clear neurological deficit was observed. Posterior fixation from T4 to T7 (three levels) using a combination of TSDs and PSs was performed in the lateral decubitus position for maintaining the spinal
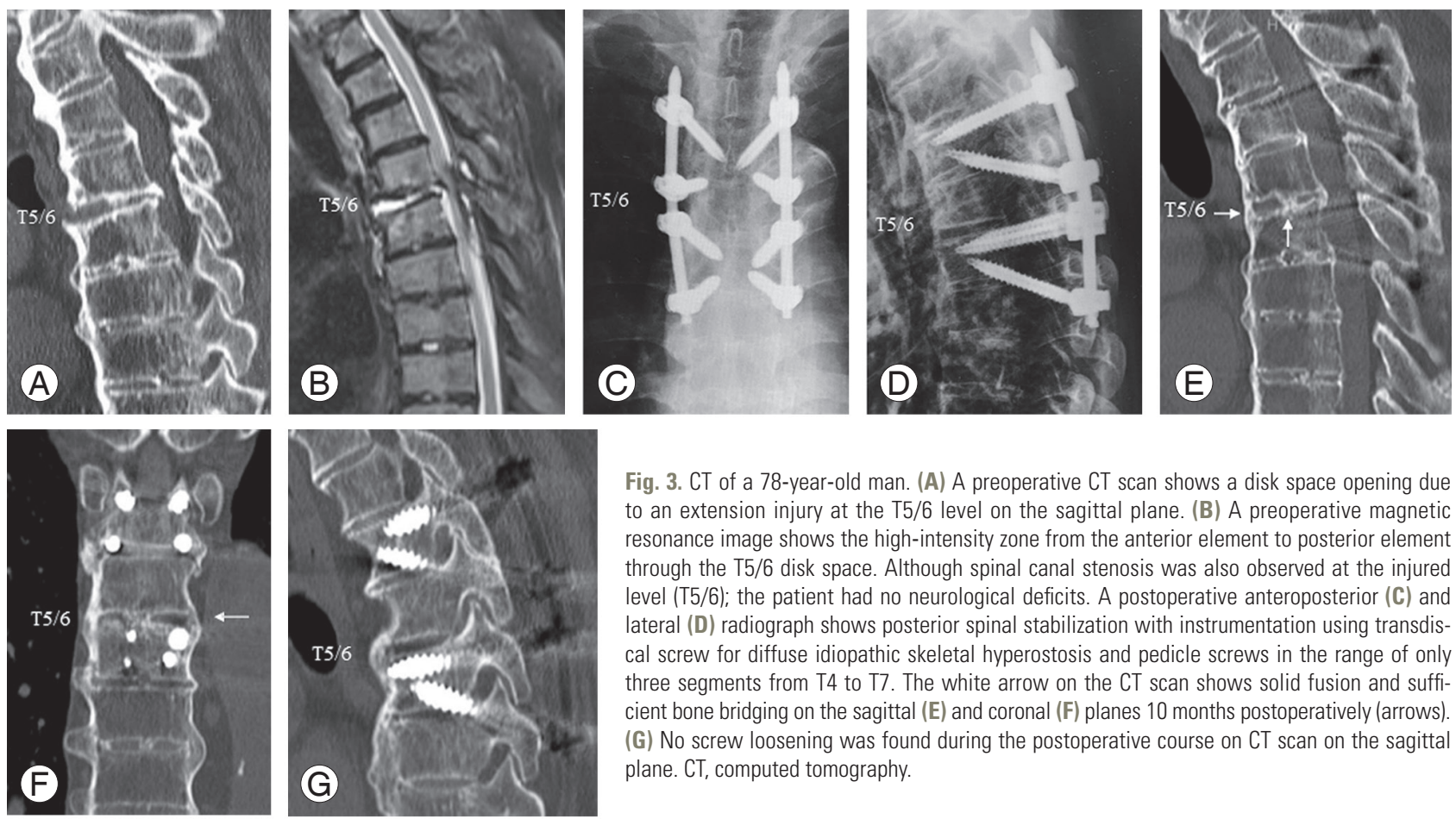

Fig. 3. CT of a 78-year-old man. (A) A preoperative CT scan shows a disk space opening due to an extension injury at the $T 5 / 6$ level on the sagittal plane. (B) A preoperative magnetic resonance image shows the high-intensity zone from the anterior element to posterior element through the $T 5 / 6$ disk space. Although spinal canal stenosis was also observed at the injured level (T5/6); the patient had no neurological deficits. A postoperative anteroposterior (C) and lateral (D) radiograph shows posterior spinal stabilization with instrumentation using transdiscal screw for diffuse idiopathic skeletal hyperostosis and pedicle screws in the range of only three segments from T4 to T7. The white arrow on the CT scan shows solid fusion and sufficient bone bridging on the sagittal (E) and coronal (F) planes 10 months postoperatively (arrows). (G) No screw loosening was found during the postoperative course on CT scan on the sagittal plane. CT, computed tomography. 
alignment. Ten months after the surgery, sufficient bone bridging and union were accomplished, and no loosening of the implants was observed. At the final follow-up (21 months after surgery), he had no back pain and no difficulties with daily living (Fig. 3).

\section{Discussion}

Spinal fracture accompanied by DISH often occurs with severe spinal instability because of three-column injury. Therefore, displacement of the fracture site can easily occur, resulting in delayed spinal injury [3]. Furthermore, the concentration of stress on the fracture site because of the long lever arm of the ankylosed spine can delay bone union and eventually cause pseudoarthrosis [7]. Thus, spinal fixation using spinal instrumentation should be performed as early as possible for spinal fractures accompanied by DISH $[1,2]$.

For PS insertion into the vertebrae accompanied by DISH, especially in elderly populations, adequate PS stability cannot be achieved because of the considerable loss of bone quality. According to Reinhold et al. [8], spinal columns accompanied by DISH, particularly in cases involving elderly populations, exhibit continuous bone bridging hyperplasia on the outside of the vertebral bodies, while cancellous bone progressively weakens inside the vertebral body. Moreover, Westerveld et al. [9] reported that stress shielding occurs in areas with ossified ligaments outside the vertebral body caused by hyperplasia and thickening. Therefore, the bone density within these vertebral bodies is often significantly lower than that within general vertebral bodies [9]. Furthermore, in spinal columns with many segments affected by ankylosing spondylitis, the lever arm becomes longer; thus, bone fracture can easily occur with trivial injury mechanisms. Therefore, the spinal instrumentation around the fracture site can create a stress concentration following spinal fixation [10]; this should be kept in mind by the spine surgeon.

Spinal instrumentation for spinal fracture accompanied by DISH is generally performed via the posterior approach rather than the anterior approach because of the aforementioned problems of the osteoporotic vertebral body and lever arm. The posterior approach offers the following advantages over the anterior approach: various fixation methods can be selected, depending on the spinal instrument used, and posterior spinal fixation can be performed simultaneously with nerve decompression [2]. Many reports investigating the use of screws have focused on PSs, with which the extent of fixation is determined by stress concentration and bone fragility and ranges from one vertebra up and one vertebra down to at least three vertebrae up and three vertebrae down. In recent years, an increasing number of studies have reported on screw pullout and loosening occurring with a fixation range of up to two vertebrae up and two vertebrae down that ultimately may cause construct failure; many of these reports have recommended posterior fixation with PSs with a fixation range of at least three vertebrae up and three vertebrae down $[11,12]$.

The TSD is characterized by the screw passing through the endplates of two adjacent vertebral bodies. Therefore, we think that TSD insertion is a specialized technique only for spinal columns accompanied by DISH, wherein the adjacent segments are ankylosed and the flexibility of the spinal column is remarkably reduced. Our results showed that the HU value of the TSD trajectory was significantly higher than that of the PS trajectory and that the maximum intraoperative screw insertion torque for TSDs was significantly higher than that for PSs. Therefore, we believe that TSDs can be a stronger anchor than conventional PSs even in cases of the osteoporotic spines.

According to anatomical studies of the vertebral endplates from the thoracic spine to the lumbar spine, the center to the anterior portion is the weakest region, while the posterolateral portion is the strongest $[13,14]$. Therefore, it is important to avoid an excessively large screw insertion angle in the axial direction and to insert the TSD along the axis of the pedicle as much as possible to enable the screw to hold the posterolateral portion of the vertebral endplate.In recent years, there have been increasing reports of the use of the posterior approach and PPSs for minimizing the surgical invasiveness. In many of these reports, although posterior spinal fixation was performed over an extensive range of three vertebrae up and three vertebrae down, the estimated intraoperative blood loss and rate of perioperative complications were lower than those with conventional open surgery using PPSs [10]. The TSD is essentially based on the use of PPS, using which, we could reduce the surgical invasiveness with a mean surgical time and estimated intraoperative blood loss of $165.9 \pm 45.5$ minutes and $71.0 \pm 53.4 \mathrm{~mL}$, respectively. According to the literature, spinal injury accompanied by DISH often occurs in the elderly popu- 
lation with a mean age of 68-81.5 years, and the perioperative mortality rate is relatively high, at approximately $10 \%-30 \%[9,10,15]$. Caron et al. [11] performed posterior fixation for at least three vertebrae up and three vertebrae down with conventional open surgery and reported good surgical result. However, the perioperative mortality rate was approximately $30 \%$, and they experienced surgical wound complications in $12 \%$ of the patients. Our patients also represented an elderly population with a mean age of $81.2 \pm 7.5$ years; one patient $(7.6 \%)$ died after surgery due to preexisting heart disease. The mortality rate remained high even though a minimally invasive technique was used for these elderly patients. Therefore, careful attention is required for the successful treatment of these patients.

TSD can pass through the disk space to reach the adjacent vertebral body; therefore, the adjacent vertebral body is simultaneously fixed with one screw. Thus, by inserting the TSD continuously into the adjacent vertebral body, total four screws can be inserted into the cranial adjacent vertebral body. Two screws from the caudal adjacent vertebrae are inserted into the anterior column and two other screws from the involved vertebrae are inserted into the posterior to the middle columns. This can be considered a characteristic feature of the TSD. This means that the local stability of craniocaudal level around the fracture site of the spine can be secured even with a small number of spinal instruments. Therefore, compared to conventional posterior fixation with PSs, TSDs has the potential to reduce the number of spinal instruments per case (Fig. 1). This ability of the TSD can help decrease the surgical invasiveness and mortality rate. Although there is a possibility that the local stress may increase by the insertion of four screws for one vertebral body; we believe that TSD inserted into the craniocaudal vertebrae around the involved vertebra can decrease this local stress. If there is no vertebral endplate injury at the level of vertebral body fracture, this endplate can be used for TSDs.

This study has certain limitations. The relatively low incidence of spinal injury accompanied by DISH in clinical practice resulted in the small sample size in this study. In the future, additional examinations are warranted with a larger study population and longer follow-up periods. Furthermore, as our biomechanical study only examined the maximum intraoperative screw insertion torque, further studies might be warranted to examine the TSD pullout strength, the strength of a construct with a rod connected, and stress loading to the adjacent segments. The combination of TSDs and PSs was evaluated in this study. When we started to use TSD, the claw-like configuration with combination TSD and PS was much better for pullout strength as screws and rods construct. However, as a result of our evaluation of the HU measurement of the screw trajectory and the screw insertional torque during the surgery, there is a potential that all TSDs construct can be recommended, and this issue requires continuous investigation in the future. However, we found that TSDs can be a stronger anchor than conventional PSs for fractures of the thoracolumbar spine accompanied by DISH, especially in elderly patients with severe osteoporosis.

\section{Conclusions}

We reported on the surgical results of 13 elderly patients who underwent posterior fixation using TSDs combined with PPSs for the treatment of fracture of the thoracolumbar spine accompanied by DISH and performed a biomechanical study of TSDs. Better surgical results were achieved using TSDs combined with PPSs for posterior fixation, and utilizing PPSs with TSDs could reduce the surgical invasiveness. The HU value of the screw trajectory and the maximum intraoperative screw insertion torque were significantly higher for TSDs than for PSs. These results could suggest that TSDs could be a stronger anchor than PSs for spinal columns with low bone density and a loss of flexibility because of DISH.

\section{Conflict of Interest}

No potential conflict of interest relevant to this article was reported.

\section{References}

1. Okada E, Tsuji T, Shimizu K, et al. CT-based morphological analysis of spinal fractures in patients with diffuse idiopathic skeletal hyperostosis. J Orthop Sci 2017;22:3-9.

2. Rustagi T, Drazin D, Oner C, et al. Fractures in spinal ankylosing disorders: a narrative review of disease and injury types, treatment techniques, and outcomes. J Orthop Trauma 2017;31 Suppl 4:S57-S74.

3. Yamamoto T, Kobayashi Y, Ogura Y, Takahashi Y, Shinozaki Y, Ogawa J. Delayed leg paraplegia associated with hyperextension injury in patients with 
diffuse idiopathic skeletal hyperostosis (DISH): case report and review of the literature. J Surg Case Rep 2017;2017:rjx040.

4. Jia H, Lubetkin EI. The impact of obesity on healthrelated quality-of-life in the general adult US population. J Public Health (Oxf) 2005;27:156-64.

5. Matsukawa K, Abe Y, Yanai Y, Yato Y. Regional Hounsfield unit measurement of screw trajectory for predicting pedicle screw fixation using cortical bone trajectory: a retrospective cohort study. Acta Neurochir (Wien) 2018;160:405-11.

6. Minamide A, Akamaru T, Yoon ST, Tamaki T, Rhee JM, Hutton WC. Transdiscal L5-S1 screws for the fixation of isthmic spondylolisthesis: a biomechanical evaluation. J Spinal Disord Tech 2003;16:144-9.

7. Hasegawa K, Takahashi H, Iida Y, et al. Spontaneous symptomatic pseudoarthrosis at the 12-13 intervertebral space with diffuse idiopathic skeletal hyperostosis: a case report. Case Rep Orthop 2013;2013:497458.

8. Reinhold M, Knop C, Kneitz C, Disch A. Spine fractures in ankylosing diseases: recommendations of the spine section of the German Society for Orthopaedics and Trauma (DGOU). Global Spine J 2018;8:56S-68S.

9. Westerveld LA, Verlaan JJ, Oner FC. Spinal fractures in patients with ankylosing spinal disorders: a systematic review of the literature on treatment, neurological status and complications. Eur Spine J 2009;18:145-56.

10. Yeoh D, Moffatt T, Karmani S. Good outcomes of percutaneous fixation of spinal fractures in ankylosing spinal disorders. Injury 2014;45:1534-8.

11. Caron T, Bransford R, Nguyen Q, Agel J, Chapman J, Bellabarba C. Spine fractures in patients with ankylosing spinal disorders. Spine (Phila Pa 1976) 2010;35:E458-64.

12. Werner BC, Samartzis D, Shen FH. Spinal fractures in patients with ankylosing spondylitis: etiology, diagnosis, and management. J Am Acad Orthop Surg 2016;24:241-9.

13. Grant JP, Oxland TR, Dvorak MF. Mapping the structural properties of the lumbosacral vertebral endplates. Spine (Phila Pa 1976) 2001;26:889-96.

14. Xavier F, Jauregui JJ, Cornish N, et al. Regional variations in shear strength and density of the human thoracic vertebral endplate and trabecular bone. Int J Spine Surg 2017;11:7.

15. Westerveld LA, van Bemmel JC, Dhert WJ, Oner FC, Verlaan JJ. Clinical outcome after traumatic spinal fractures in patients with ankylosing spinal disorders compared with control patients. Spine J 2014;14:72940. 